\title{
SESGOS DE RAZONAMIENTO Y RETÓRICA PSI EN LA TERAPIA ALTERNATIVA FLORAL
}

\author{
REASONING BIASES AND PSY RHETORIC IN \\ FLORAL ALTERNATIVE THERAPY
}

\author{
Juan Antonio González de Requena Farré* \\ Universidad Austral de Chile \\ Valdivia-Chile
}

Felipe Munoz-Rubke

Universidad Austral de Chile

Valdivia-Chile

Recibido abril de 2020/Received April, 2020

Aceptado septiembre de 2020/Accepted September, 2020

\begin{abstract}
RESUMEN
En las últimas décadas, la popularidad de terapias alternativas como las flores de Bach ha aumentado significativamente. Debido a que cualquier efecto de la terapia floral no es superior al conseguido mediante placebo, parece resultar decisiva la utilización de distintas estrategias retóricas y técnicas de persuasión. El presente estudio cualitativo buscó describir los sesgos de razonamiento y estrategias comunicativas que sustentan la justificación subjetiva de la terapia floral. Se realizó una codificación abierta y flexible de los enunciados de los participantes del blog de una terapeuta floral. Como resultado, se identificaron estrategias referidas a la personalización y autorreferencia discursiva, el saber idiográfico, la psicologización de la terapia, el error de atribución fundamental, la esencialización y el pensamiento animista. Otras estrategias incluyeron el uso de lenguaje paraterapéutico y el léxico seudocientífico, la relativización de las opciones terapéuticas, el holismo terapéutico, así como la racionalización de la esperanza, la construcción de historias explicativas y la confirmación retrospectiva. Finalmente, se discute la posibilidad de que la eficacia de estas estrategias esté dada por un excesivo psicologismo (o retórica psi), en un ambiente que favorece la posverdad.
\end{abstract}

Palabras Clave: Flores de Bach, Terapias Alternativas, Sesgos, Estrategias Comunicativas, Psicologismo.

\begin{abstract}
In recent decades, the popularity of alternative therapies such as Bach flowers has increased significantly. Since any effect of floral therapy is not superior to those achieved by a placebo treatment, the use of different rhetorical strategies and persuasion techniques seem to be decisive. The present qualitative study sought to describe the reasoning biases and communicative strategies that support the subjective justification of floral therapy. An open and flexible coding of the statements made by the participants of a floral therapist's blog was used. As a result, strategies related to personalization and discursive self-reference, idiographic knowledge, psychologization of therapy, fundamental attribution error, essentialization, and animistic thinking were identified. Other strategies included the use of paratherapeutic language and pseudo-scientific lexicon, relativization of therapeutic options, therapeutic holism, as well as rationalization of hope, construction of explanatory stories and retrospective confirmation. Finally, it is discussed that the possibility of effectiveness of these strategies is based on an excessive psychologism (or psy rhetoric), in an environment that favors post-truth.
\end{abstract}

Key Words: Bach Flowers, Alternative Therapies, Biases, Communicative Strategies, Psychologism.

\footnotetext{
*Autor correspondiente / Corresponding author: jgonzalez@spm.uach.cl
} 


\section{INTRODUCCIÓN}

$\mathrm{Al}$ enfrentar problemas de salud física y mental, existe más de una alternativa, o incluso la opción de complementar diversas terapias para lograr un tratamiento supuestamente más integral. Podríamos seguir los procedimientos recomendados por profesionales formados bajo los principios de la medicina convencional basada en la evidencia. Estas prácticas de salud sustentan las decisiones clínicas en el conocimiento obtenido mediante la investigación experta y los ensayos clínicos controlados. Así pues, los tratamientos consisten en procedimientos y fármacos que causan de modo confiable mejoría en los diversos síntomas y condiciones (Lack y Rosseau, 2016, p. 176). Otra posibilidad es seguir las indicaciones otorgadas por practicantes de la medicina alternativa, un área heterogénea que ha adquirido creciente popularidad en las últimas décadas. Entre las terapias alternativas se cuentan sistemas médicos integrativos (como la homeopatía, la naturopatía o las flores de Bach), tratamientos psicosomáticos (como la meditación o la arteterapia), prácticas corporales (como los tratamientos quiroprácticos o los masajes) y terapias energéticas (Reiki, acupuntura, terapia electromagnética). Estas terapias alternativas suelen recurrir a productos presuntamente naturales o a prácticas de control mental o psicosomático (Lack y Rosseau, 2016, pp. 178-179).

En el presente estudio nos centramos en la terapia alternativa llamada flores de Bach (Bach flower remedies). Las flores de Bach fueron desarrolladas en el siglo XX por Edward Bach, médico, patólogo y homeópata británico. Bach pensaba que las enfermedades tenían una base espiritual y que su cura no estaba asociada a la mera mejoría de síntomas físicos. Para Bach, la cura también requería de un profundo proceso de mejoría emocional (Mantle, 1997). Bach creyó identificar 38 preparaciones florales que promovían la curación de 38 estados negativos (Mantle, 1997), los cuales organizó dentro de siete categorías psicológicas: temor, incertidumbre, insuficiente interés en las circunstancias actuales, soledad, hipersensibilidad a influencia e ideas, desaliento o desesperación, así como preocupación excesiva por el bienestar de otros (Bach, 1936). Según Bach, las esencias florales eran capaces de restituir el balance y la armonía cuando las personas sufrían de problemas de personalidad o del estado de ánimo. Bach estaba muy interesado en que su sistema de curación emocional fuera entendible por todos, por lo que no concibió un proceso formal de entrenamiento para los practicantes de su disciplina (Bach, 1936).

La creciente popularidad de esta medicina alternativa no concuerda con la información disponible respecto de su utilidad para tratar de problemas de salud. Partiendo de la premisa de que el peso de la evidencia está en quienes promueven su uso, las escasas investigaciones que sugieren evidencia a favor de su eficacia están plagadas de errores de diseño, muestreo o análisis estadístico.

Callís Fernández (2011) llevó a cabo una intervención con flores de Bach en 48 escolares con hiperactividad. En dicho estudio, la autora no describió qué características tuvieron los participantes para ser considerados como "escolares con hiperactividad"; no incluyó un grupo control y solo calculó estadísticas descriptivas para dar cuenta de cambios en los síntomas de los participantes. A pesar de estas notables limitaciones, la autora concluyó que:

...la terapia floral de Bach es un tratamiento efectivo para disminuir o minimizar las manifestaciones clínicas del trastorno por déficit de atención e hiperactividad (TDAH) en la infancia [...] por todo lo anterior se recomienda incorporar la mencionada terapia como una forma más para tratar a niños con TDAH (p. 5).

La investigación dirigida por Resende, Costa, Gardona, Araújo, Mundim y Costa (2014) sugirió que las flores de Bach son eficaces en el control de factores de riesgo asociados a enfermedades cardiovasculares en ratas. Para los autores, su investigación representaba una demostración de que las flores de Bach generan cambios de índole biológica. Sin embargo, la investigación de Resende et al. (2014) presentó severas limitaciones. El estudio solo consideró 18 ratas separadas en tres grupos de seis individuos, tamaño muestral que hace difícil interpretar cualquier resultado. Además, los autores no reportaron que los tres grupos hayan tenido niveles previos similares a la intervención, en cada una de las medidas biológicas de interés. A estos errores se sumó una deficiente sección de resultados, en que los autores no incluyeron los valores de los estadísticos ni de los p-values asociados a los procedimientos utilizados (i.e., ANOVA y Tukey's HSD). De igual manera, el hecho que los autores no hayan reportado medidas de dispersión 
ni presentado ningún tipo de gráfico hace difícil evaluar la validez de sus resultados.

Algunos estudios han mostrado que las flores de Bach no son más eficientes que el placebo en el tratamiento de la ansiedad (Walach, Rilling, y Engelke, 2001) ni del trastorno de déficit de atención e hiperactividad en niños (Pintov, Hochman, Livne, Heyman, y Lahat, 2005). Aunque ambos estudios incluyeron un grupo control y un procedimiento de doble ciego, el tamaño de las muestras de ambos estudios fue relativamente pequeño (61 y 40 participantes, respectivamente). Los resultados de estos estudios son consistentes con artículos de revisión que sintetizaron la investigación disponible a la fecha. En particular, tres artículos destacaron que la evidencia actual sugiere que las flores de Bach no resultan más eficientes que el placebo en el tratamiento de distintas condiciones de salud (Ernst, 2010; Halberstein, Sirkin, y Ojeda-Vaz, 2010; Thaler, Kaminski, Chapman, Langley, y Gartlehner, 2009).

Si bien pocos estudios han evaluado los efectos del tratamiento floral, la evidencia disponible indica que no existe razón alguna para creer que las flores de Bach resultan terapéuticamente beneficiosas. En la medida en que no se basan en la evidencia, las terapias complementarias alternativas dan cuenta de la presencia de un efecto placebo; esto es, de una mejoría aparente en la salud sin un tratamiento efectivo. El placebo concierne a la percepción subjetiva de mejoría más que a la evidencia objetiva obtenida mediante pruebas confiables, aunque puede tener efectos psicológicos, conductuales u fisiológicos asociados (Lack y Rosseau, 2016, pp. 180-183). En ese sentido, el efecto placebo de la terapia floral se vincula a las creencias y expectativas personales, a la apertura e inquietud espiritual de los consultantes -incluida la disposición al pensamiento positivo-, y operaría en una dimensión aparentemente intangible e inexplicable (Howard, 2007). El efecto subjetivo de las terapias alternativas depende de estrategias retóricas y técnicas persuasivas como las siguientes: la promesa de cura para todos los males; el empleo de fórmulas que apelan a remedios ancestrales, esencias secretas o productos únicos; la jerga aparentemente médica; el tono defensivo ante la medicina convencional y las instituciones de salud; el relato de casos sorprendentes; finalmente, el carácter exclusivo del tratamiento, únicamente disponible por un medio o persona (Lack y Rosseau, 2016, p. 188). También es importante reconocer estrategias de comunicación y divulgación de las terapias alternativas, como el enmarcado discursivo y la vinculación temática con todo un discurso de la felicidad, el cual circula profusamente en Internet, se difunde masivamente en las redes sociales, está fácilmente disponible en los buscadores, y llega individualmente al usuario personal de las plataformas, sin contrapeso crítico alguno (López Cantos, 2017a, 2017b).

Como contribución al abordaje cualitativo de las terapias alternativas complementarias, este estudio se propone precisamente describir las estrategias retóricas y técnicas persuasivas que sustentan el efecto placebo y, por tanto, el significado subjetivo atribuido a la participación en la terapia floral.

\section{METODOLOGÍA}

Para reconstruir las significaciones subjetivas que emanan de la participación en una terapia alternativa, este estudio apuesta por un enfoque cualitativo. No en vano, los métodos cualitativos se hacen cargo típicamente de la descripción de las expresiones y discursos de las personas, de manera inductiva, tratando de comprenderlos en su propio marco de referencia (Taylor y Bogdan, 1994, p. 20).

Este estudio es descriptivo y transeccional, pues solo considera la caracterización de cierto discurso terapéutico alternativo tal como aparece registrado en el momento de la investigación.

\section{Muestra}

El estudio contempla un corpus textual conformado por los contenidos de un blog de la terapia alternativa conocida como flores de Bach (Bach, 1936). En particular, hemos recogido un repertorio de textos del blog Gotas de Bach, cuya dirección electrónica es http://www.gotasdebach. $\mathrm{com} /$. Su autora es la terapista floral española Anna Selva. Específicamente, se abordan los contenidos albergados en las siguientes secciones de este blog, publicadas entre el 2010 y 2018: “¿Quién soy?”; "Las enfermedades físicas y las emociones”; “ ¿Por qué un Estudio Previo antes de tomar Flores de Bach?", "Las 38 Flores de Bach"; "Catalizadores Florales de Bach"; "Emociones a tratar con Flores de Bach"; "Las esencias Áureas y las Flores de Bach"; "Flores de Bach/Homeopatía"; "Terapia Floral... ¿no resulta algo largo y complicado?"; "Las Crisis Curativas de Bach"; "Formas de Reacción a las Esencias Florales"; "Flores de Bach y dependencia"; "Después del Sufrimiento... las secuelas 
emocionales en la enfermedad"; finalmente, la sección "Testimonios", que recoge interacciones discursivas en forma de consultas de supuestos pacientes y respuestas de la terapeuta (además de algunos testimonios personales de agradecimiento).

Adicionalmente, para contrastar los patrones de interacción comunicativa del blog mencionado y los de la medicina convencional basada en la evidencia, se recogieron las interacciones comunicativas en línea entre médicos y pacientes de un foro virtual de salud publicado en la siguiente dirección: https://salud.ccm.net/forum/affich-7111-consultas-medicas-en-linea-gratis. Concretamente, se consideraron 60 entradas de dos doctores distintos y de sus respectivos consultantes. De las interacciones comunicativas registradas tanto en el blog de terapia floral (en la sección "Testimonios") como en el foro virtual de medicina convencional, hemos analizado aquellas que responden a un mismo género de discurso que cabría considerar como consulta a un experto. Se trata un tipo de interacción comunicativa caracterizado por la búsqueda de información, en la cual alguien que no dispone del saber preciso formula una pregunta al especialista, y portador de un supuesto saber u opinión experta se propone desarrollar una posición informada, propagar su conocimiento e influir al interlocutor lego; de ese modo, el consultante podría salir críticamente de su posición de ignorancia, alcanzaría una conclusión razonable bajo la forma de una respuesta plausible y, así, estaría en condiciones de adoptar medidas al respecto (Walton, 1999, pp. 44-45; Walton y Krabbe, 2017, pp. 115-117).

La decisión de trabajar con este tipo de corpus se sustenta en la oportunidad que brinda para disponer de interacciones discursivas transcritas que recogen las experiencias y expectativas de las personas involucradas en esta clase de terapias. De acuerdo con los criterios de calidad de un estudio de carácter cualitativo de la investigación, el corpus ilustra de modo fidedigno este tipo de discursos, y los textos analizados han sido publicados por la propia fuente; además, el repertorio de textos permite una descripción e interpretación en profundidad del desempeño discursivo, de modo que el estudio puede transferirse de modo verosímil a discursos semejantes.

\section{Procedimiento de análisis}

Para llevar a cabo una descripción de las significaciones subjetivas, se procedió a la codificación abierta y flexible de los enunciados de los participantes. De esa manera, se pretendía especificar las características descriptivas de estos discursos y de conceptualizar sus categorías descriptivas más relevantes, por medio de una comparación constante -teóricamente informada- de los enunciados de los participantes (Strauss y Corbin, 2002, pp. 110-133).

En los enunciados de los distintos participantes se identificaron distintas categorías de sesgo de razonamiento que permiten dar cuenta del sustento subjetivo de estos discursos, así como de la reproducción de la confianza y las expectativas en torno a esas terapias. Aunque existen repertorios de sesgos cognitivos definidos en la literatura de la psicología social (Pohl, 2004; Sutherland, 1992) y de falacias argumentativas planteadas por la teoría de la argumentación (Hamblin, 2016; Tindale, 2007; Vega Reñón, 2013; Walton, 1999), este estudio optó por caracterizar inductivamente los modos emergentes de autojustificación subjetiva de los discursos terapéuticos alternativos. De todos modos, en la descripción y comparación teórica constante se emplearon algunas conceptualizaciones de los sesgos cognitivos, errores de razonamiento y sesgos argumentativos disponibles en la bibliografía especializada.

Desde que Tversky y Kahneman (1974) introdujeron la noción de sesgo cognitivo para caracterizar ciertos errores sistemáticos en nuestros juicios acerca de probabilidades, distorsiones atribuibles a las propias heurísticas o procedimientos de búsqueda del razonamiento humano (como el atenerse a la evidencia más disponible, esto es, más presente, llamativa, vívida o impactante; o bien el realizar estimaciones a partir de lo que consideramos típico de una categoría de entidades), en psicología social se ha extendido la referencia a ilusiones cognitivas sistemáticas e involuntarias que afectan a procesos cognitivos como el juicio o el razonamiento (Pohl, 2004, pp. 2-3). Así pues, los sesgos cognitivos cubren no solo las ilusiones derivadas de una apreciación o estimación inadecuada de la evidencia, sino también ciertos errores en el razonamiento lógico o la inferencia estadística (Pohl, 2004, pp. 3-6). Por otra parte, las falacias argumentativas han sido abordadas desde distintas perspectivas: se puede caracterizar la falacia en sentido lógico y atender al argumento textual como producto cuya validez probatoria fallida puede determinarse con base en criterios lógicos o epistemológicos; es posible establecer la falacia en sentido dialéctico, esto es, 
en relación con los procedimientos de interacción argumentativa y las normas del debate razonable y la discusión racional; por último, existe un acercamiento retórico a la falacia como manipulación, el cual se centra en los procesos de comunicación y de influencia en el auditorio, mediante estrategias argumentativas eficaces para lograr un discurso persuasivo (Vega Reñón, 2013, pp. 107-118). En cierto sentido, es admisible hablar de sesgos en la argumentación para referirse a las desviaciones en la interacción dialógica, atribuibles al empleo contextual de los argumentos para ciertos fines comunicativos o a la credibilidad del interlocutor, más que solo a la relación lógica entre premisas y conclusión. Este tipo de sesgos argumentativos se opone a la objetividad, imparcialidad y crítica; introduce desequilibrios en el intercambio dialógico o cierra unilateralmente la argumentación, y, así, puede afectar a la credibilidad racional de nuestros argumentos y, eventualmente, conducir a errores, engaños o falacias (respecto a esta noción de bias in argumentation véase Walton, 1999, cap. 3).

Ciertamente, la relación entre los sesgos cognitivos y las falacias argumentativas no es obvia, y en algunos enfoques de la lógica informal se distingue entre falacias y errores de razonamiento, del mismo modo que se remarca escrupulosamente la diferencia entre el razonamiento o la inferencia -en tanto que procesos psicológicos de transformación o elaboración de representaciones o creencias- y, por otra parte, la argumentación, como práctica comunicativa y, por tanto, social en que se produce un texto con el propósito de razonar discursivamente algo para persuadir al destinatario (véase Marraud, 2013, cap. 1). No obstante, otros planteamientos en teoría de la argumentación definen el razonamiento a partir de la inferencia, y el argumento desde el razonamiento, como si el argumento tuviese simultáneamente un aspecto de razonamiento inferencial $\mathrm{y}$ otro aspecto discursivo: si la inferencia es el paso de una premisa a una conclusión, el razonamiento consistiría en un encadenamiento de inferencias, en el cual alguna conclusión inferencial sirve de premisa para otra inferencia; asimismo, el argumento consistiría en un conjunto estructurado de inferencias o en una cadena de razonamientos con determinados propósitos y funciones discursivas, para resolver alguna cuestión en el curso del diálogo (Marraud, 2007, pp. 27-29; Walton, 1999, pp. 27-29). Por lo demás, la frontera entre los aspectos comunicativos y cognitivos del discurso es sumamente porosa, problemática y productiva: por un lado, la pragmática contemporánea ha destacado los aspectos cognitivos de la comunicación humana, que involucra no solo la acción intencional, el código lingüístico y la interacción social, sino también representaciones mentales, razonamiento acerca de representaciones y procesos inferenciales (Escandell Vidal, 2014; Wilson y Sperber, 2004); por otro lado, la psicología discursiva y retórica nos ha recordado que las representaciones mentales y los estados cognitivos se inscriben en prácticas comunicativas cotidianas, interacciones conversacionales, formas de hablar, relatos y tradiciones de argumentación (Potter, 1998, pp. 136-140; Shotter, 2001, pp. 52-56).

En este trabajo no pretendemos resolver el dilema teórico de si acaso la argumentación es una actividad lingüística con aspectos cognitivos, que habría que estudiar desde las ciencias del lenguaje, o bien se trata de una actividad cognitiva con expresión discursiva, que habría que estudiar desde las ciencias cognitivas (Plantin, 2011, p. 29). Al fin y al cabo, en la historia de la concepción de las falacias han coexistido una tradición discursiva centrada en la identificación y evaluación normativa de falacias usuales en contextos argumentativos y, por otro lado, una tradición cognitiva interesada en la descripción y explicación de las falacias como errores o sesgos cognitivos (Vega Reñón, 2013, pp. 59-61). Incluso, en algunos autores, la concepción estándar de la falacia parecía incorporar aspectos lógicos y psicológicos: "En el estudio de la lógica, se acostumbra reservar el nombre de 'falacia' a aquellos razonamientos que, aunque incorrectos son psicológicamente persuasivos" (Copi, 1984, p. 81). En todo caso, la descripción de sesgos cognitivos y sesgos en la argumentación en un contexto discursivo acotado tal vez pueda alumbrar algunas afinidades electivas entre ciertas falacias argumentativas, errores de razonamiento y patrones comunicativos.

Para detectar los errores de razonamiento y sesgos argumentativos asociados a la autojustificación subjetiva se atendió a las estrategias de legitimación del terapeuta. Para establecer el tipo de interacción personal que suscita la consulta terapéutica alternativa en línea, se realizó una reconstrucción estructural del patrón típico de los movimientos comunicativos o retóricos más recurrentes, y se contrastó con la pauta de movimientos retóricos de la consulta médica estándar en línea. El concepto 
de movimiento retórico se emplea en la presente investigación con el sentido técnico en que lo utiliza el análisis de géneros propuesto por Swales: como "una unidad retórica o discursiva que desempeña una función comunicativa coherente en un discurso escrito o hablado" (Swales, 2004, p. 228), de modo que cada movimiento comunicativo puede realizarse de manera flexible en distintas formas lingüísticas.

\section{RESULTADOS}

\section{Estrategias de autopresentación y autojustificación de la persona del terapeuta}

En el discurso de la terapeuta floral, se reconocieron ciertos tópicos argumentativos -sustentados en ciertos sesgos identificables-que permiten el posicionamiento y la autorización del sujeto de discurso. Los principales tópicos en la legitimación del lugar de la terapeuta conciernen a la justificación de su saber, al carácter especial de su oficio, su trayectoria personal y su profunda capacidad terapéutica para resolver algunos de los problemas más arraigados de la existencia humana. Mediante esa apelación a un saber especial y personal, la terapeuta busca investirse de autoridad como sujeto de la enunciación y, así, garantizar sus pretensiones de validez. A continuación, desglosamos el repertorio de los sesgos cognitivos y argumentativos evidenciados en el discurso autolegitimador de la terapeuta.

\section{Personalización y autorreferencia}

La justificación del saber de la terapeuta no se basa en la presentación de formación y especialización profesional o académica. En cambio, se sustenta en cierto camino de vida, en una experiencia de iniciación, en características personales y cualidades innatas (por ejemplo, su empatía y cercanía personal) e, incluso, en su "memoria celular de otras vidas" (Selva, “¿Quién soy?”, párrafo siete). La legitimación de su saber terapéutico se vincula a una apuesta existencial y a un compromiso decidido por asumir tanto la propia vocación como las necesidades subjetivas y la autoexpresión espontánea de las cualidades internas.

A diferencia de las profesiones relacionadas con la salud basadas en un currículum académico, una estructura disciplinar y un nicho profesional legalmente reconocido, la terapeuta floral apela a la expresión subjetiva y a la vocación personal como legitimaciones de su ejercicio, el cual concibe como un modo de vida y un propósito vital ("¿Quién soy?", párrafo diez). Aunque menciona los escritos del precursor de la terapia floral (Bach, 1936), la terapeuta recomienda a sus consultantes -autorreferencialmente- los contenidos de su propia página, como autora de todos ellos y escritora tan solo de aquello que ella misma ha experimentado ("¿Quién soy?", párrafo dieciocho). Desde esa perspectiva, el discurso de la terapeuta se justifica desde una posición de sujeto autorreferencial e intensamente personal, no muy concordante con los hábitos de impersonalidad, imparcialidad y crítica objetiva que caracterizan a nuestras tradiciones científicas y a nuestra cultura pública. La presencia de compromisos vocacionales y confesionales con una posición determinada constituye un indicador de propensión al sesgo argumentativo y a la argumentación unilateral, sobre todo si el hablante tiene algo que ganar con su posición, y pone precio a su palabra y sus argumentos (Walton, 1999, pp. 91-99).

\section{El saber idiográfico de una egoterapia}

No solo la posición de sujeto en el discurso de la terapeuta se justifica como una singularidad; también el objeto o tema de la terapia floral se presenta como algo que concierne a la naturaleza interior de cada persona individual, en tanto que ser único con una experiencia singular incomparable. En la perspectiva de la terapeuta, solo profundizando en la singularidad interior de cada persona se puede establecer el origen del problema y el tratamiento adecuado para las necesidades de cada individuo. La terapeuta apela a un saber que no es nomotético ni nomológico, pues no se basa en la cobertura explicativa y la predicción fenoménica de los hechos, sino en la comprensión empática de cada personalidad singular; esto es, en un saber estrictamente idiográfico (por utilizar la distinción forjada en el debate decimonónico acerca de estatuto epistemológico de las Geisteswissenschaften o ciencias del espíritu).

A diferencia del marco metodológico de los saberes hermenéuticos basados de la interpretación del sentido de los acontecimientos, este tipo de saber terapéutico no presupone horizontes comunes de significación o comprensiones tradicionales compartidas, ya que solamente apela a la comprensión interior de la personalidad y a la conexión empática entre individuos inconmensurables. Así, la terapeuta insiste convenientemente con cada consultante en la necesidad de un estudio personalizado previo para 
enfrentar la terapia individual (Selva, “¿Por qué un Estudio Previo antes de tomar Flores de Bach?", párrafos siete y siguientes). Desde su perspectiva, la terapia floral es un saber que tiene por objeto al individuo singular e inconmensurable; constituye una terapia absolutamente individualizada y muy bien sintonizada con la sensibilidad terapéutica de nuestro tiempo. Ahora bien, el tratamiento de casos comparables como si fueran esencialmente diferentes constituye un ejemplo de argumentación sesgada y unilateral, que afecta a las pretensiones de validez (Walton, 1999, pp. 106-107).

\section{Psicologización de la terapia y error de atribución fundamental}

En un marco epistemológico que tiene por sujeto del saber a una persona especial y asume como objeto la individualidad singular, prácticamente todos los problemas que involucran condiciones externas $\mathrm{y}$ variables objetivables pueden interpretarse como problemas derivados, en última instancia, de la interioridad psíquica, de la historia individual y de la personalidad singular. La terapeuta floral considera de modo reiterado que los desequilibrios interiores y las alteraciones anímicas dan cuenta de muchos malestares físicos y enfermedades corporales, pero también de la mayoría de los problemas interpersonales y de relación con el exterior (Selva, "Las enfermedades físicas y las emociones"). Entre los aspectos que se pueden tratar floralmente, la terapeuta incluye una gama de emociones negativas -como los temores y la tristeza-, los traumas sobrellevados por mucho tiempo y la pérdida de motivación e ilusión. Asimismo, habría un remedio floral para problemas sociales o interpersonales como las rupturas sentimentales, la soledad o la dificultad para relacionarse con los otros (Selva, "Emociones a tratar con Flores de Bach"). Incluso los malestares externos y dolencias físicas podrían tratarse floralmente -según la terapeuta-, especialmente aquellos para los cuales no existe una explicación médica evidente (Selva, "Las enfermedades físicas y las emociones", último párrafo).

La terapia floral permite tratar casi todo, en la medida en que no hay ámbito de la existencia humana que resulte ajeno a los estados emocionales y la vida psíquica interior. La psicología social caracteriza como error de atribución fundamental esta tendencia a sobrestimar la importancia de los factores relacionados con la personalidad, en desmedro de las influencias contextuales o ambientales
(Aronson, 2000, pp. 162-165). En el caso de la terapia floral, el error de atribución fundamental parece asumir la forma de una psicologización general de las problemáticas humanas.

\section{Esencialización mistificadora y pensamiento animista}

Bajo un marco epistemológico de sujetos y objetos absolutamente singulares e inconmensurables, en la terapia floral se perfila una ontología en que no rigen las propiedades objetivas y relaciones calculables de entidades con condiciones de irrupción determinadas, relativamente independientes del observador. La decisión ontológica fundamental de la terapia floral consiste en la invocación de esencias íntimas y cualidades inherentes, cuya profundidad interior no se agota en las propiedades aparentes. Cada una de las 38 flores que constituyen el repertorio de la terapia de Bach se caracteriza por una esencia profunda relacionada con la esencia interna de algún estado anímico, desequilibrio emocional, tipo de personalidad, motivación existencial o meta espiritual (Selva, "Las 38 flores de Bach").

Este universo de correspondencias esenciales singulares responde a cierta entificación metafórica (Lakoff y Johnson, 1998, pp. 63-67), al atribuir a nuestras experiencias e interacciones el carácter profundo de sustancias o entidades discretas que, a pesar de su esencia interior insondable, se pueden aislar y dosificar. También es importante reconocer en este esencialismo generalizado un ejercicio de mistificación terapéutica $-y$ determinado fetichismo de lo singular-, en virtud del cual se otorga un extraño poder sobrenatural a la cosificación de un repertorio de condiciones sociales y de relaciones interpersonales (Rossi-Landi, 1980, pp. 197-199). En la terapia floral, la entificación de un repertorio de esencias internas parece asociarse a cierta convicción animista de que los principales factores y condiciones causales en nuestra relación con el mundo son las emociones, las correspondencias emocionales y los equilibrios anímicos internos.

\section{Lenguaje paraterapéutico y léxico seudocientífico}

El universo epistemológico y ontológico de la terapia floral guarda una relación ambivalente con las disciplinas terapéuticas y las ciencias de la salud. Para legitimar el carácter especial y singular de las flores de Bach, la terapeuta se distancia del léxico de la terapia, pues considera que connota 
algo diferente a la simple ingesta de mezclas de esencias florales como ayuda para superar obstáculos emocionales. La terapia floral no tiene nada de farmacopea artificial ni de intervención externa, ya que solo consistiría en un complemento espiritual sencillo y natural -una "ayuda extra" - en el curso de un proceso personal de transformación interior (Selva, "Terapia Floral... ¿no resulta algo largo y complicado?").

Por otra parte, la terapeuta floral recurre a un lenguaje paraterapéutico en que se hace presente el léxico de la enfermedad y el tratamiento, los síntomas y la curación, la dosificación de los preparados y las reacciones curativas (Selva, "Las Crisis Curativas de Bach"). Pese al compromiso de la terapeuta con los procesos de autotransformación interior, las descripciones de la eficacia terapéutica de las flores de Bach recurren a un vocabulario con connotaciones fisicoquímicas y ocasionalmente seudobiológicas. Las esencias florales son caracterizadas como "puras y armoniosas vibraciones de energía" (Selva, "Formas de Reacción a las Esencias Florales", párrafo 6). Además, se describen ciertos "catalizadores" florales que facilitan la comunicación esencial y el desbloqueo emocional, en virtud de su potencia catalizadora para acelerar los procesos (Selva, "Catalizadores Florales Bach"), así como un "psiquismo celular" y la existencia de "frecuencias energéticas a nivel celular" sobre las cuales trabajarían las flores de Bach (Selva, "Las Esencias Áureas y las Flores de Bach", párrafo sobre las Flores del Alba). Con justa razón, se ha objetado el empleo seudocientífico del vocabulario de las ciencias naturales en humanidades y filosofía (Sokal y Bricmont, pp. 22-23), pero parece que los abusos de la jerga seudocientífica han trascendido a los ambientes de la terapia alternativa.

\section{La relativización de las opciones terapéuticas}

Como parte de la actitud ambivalente hacia las ciencias de la salud, la terapeuta floral asume una posición muy matizada ante la medicina convencional. Así, se plantea cierta relación de complementación entre los tratamientos médicos basados en fármacos y el tratamiento de autotransformación interior y búsqueda de equilibrio anímico, sustentado en las esencias florales. Esta presuposición de una doble verdad terapéutica considera que los tratamientos médicos de corte farmacológico solamente intervienen sobre los síntomas, pero dejan intocada la causa profunda de nuestros problemas, asociada a desequilibrios y alteraciones de estados anímicos internos. Además, cada tipo de tratamiento es visto como poseedor de un ritmo y temporalidad propios. Mientras la medicina opera con sustancias químicas sobre síntomas puntuales y en el corto plazo, la terapia floral asume un proceso lento y de larga duración mediante la búsqueda de la realización y el equilibrio personales. A pesar de relativizar el alcance de cada tipo de tratamiento, la terapeuta floral invoca cierto holismo en el tratamiento y recomienda la conjugación de la medicina externa y la terapia interna (Selva, "Las Enfermedades físicas y las emociones"; "Testimonios", entrada del 12 de febrero de 2018).

La legitimación de un nicho terapéutico propio para las flores de Bach no solo pasa por un reparto de competencias con la medicina tradicional, sino también requiere de una diferenciación escrupulosa de otros tipos de terapia no convencional, como la Homeopatía. A diferencia de la terapia homeopática, que trata las enfermedades con pequeñas cantidades de la misma sustancia que provoca los síntomas, la terapia floral trabajaría en el ámbito sutil de los estados emocionales: reemplaza lo negativo y lo patológico con estados emocionales positivos y amplía del equilibrio anímico. En ningún caso se pretende que la terapia floral pueda reemplazar a los otros tipos de terapia; solamente se le atribuye una misión distinta a las flores de Bach, como complemento maravilloso de las otras terapias posibles (Selva, "Flores de Bach/ Homeopatía"). En suma, la verdad terapéutica se escribe en plural desde la perspectiva de la terapeuta floral, y eso vale también para los tratamientos aconsejados por la terapeuta floral, siempre tan singulares e idiosincráticos como los problemas personales y emocionales en juego. El saber y el tratamiento florales son siempre relativos, absolutamente relativos; pero el problema epistemológico del relativismo -ya sea que apele al sincretismo o a la tolerancia indiferente- consiste en que priva de sentido a los intentos de distinguir de manera relevante lo correcto y lo falso, así como desfonda todo intento de argumentación racional con pretensiones de validez (Rescher, 1993, pp. 151-157).

\section{Holismo terapéutico y omniterapia}

La ontología y epistemología de la singularidad absoluta se asocia en la terapia floral con cierta apuesta por el holismo. No se trata solamente de que, para la terapeuta floral, todo lo que ocurre en 
nuestras vidas se encuentre relacionado en virtud de algún hilo conductor y patrón de conjunto; además, esta convicción impone una búsqueda exhaustiva de la totalidad del mundo interno del paciente y la armonización de todos los niveles de su vida emocional (Selva, “¿Por qué un Estudio Previo antes de tomar Flores de Bach?"). La terapeuta floral se hace cargo de la totalidad por medio de una perspectiva de conjunto, y así puede tratar todo tipo de padecimientos. El holismo terapéutico esboza, pues, una auténtica omniterapia, un tratamiento posible de todo, en todos los niveles y sobre el conjunto del mundo propio de cada persona singular.

La pretensión de un saber holístico que caracteriza a esta y otras terapias alternativas no sobrevive a la crítica racional. No en vano, el todo invocado por el holismo puede significar la totalidad de los componentes o aspectos de algo, pero también nos puede remitir a las características que configuran un patrón de organización o permiten describir una estructura organizada. El segundo sentido, referido a las pautas de organización de un sistema, es susceptible de estudio científico, aunque no sea posible agotar la totalidad de algo en el primer sentido, y no haya una descripción científica sin selección de propiedades (Popper, 1984, pp. 9093). El caso es que el holismo terapéutico -como otros holismos contemporáneos- parece confundir las dos acepciones de totalidad y pretende aportar un saber profundo y una intervención procesual respecto del todo del mundo interior y la esencia completa de cada paciente singular.

\section{De la racionalización de la esperanza a la confirmación retrospectiva}

Para compatibilizar con el juicio basado en la evidencia ese complejo universo epistemológico y ontológico de la terapia floral, la terapeuta hace gala de todo un repertorio de sesgos cognitivos estudiados por la Psicología social. En el discurso de la terapeuta floral se expresa reiteradamente la convicción de que el autoconocimiento personal y la armonización del estado de ánimo permiten equilibrar nuestras peripecias vitales y procuran un camino de autorrealización, ya que nada sería imposible si se vive en armonía emocional interna. Asimismo, ella argumenta que el acompañamiento espiritual personalizado del terapeuta y de un tratamiento floral individualizado impulsan una auténtica revolución interior y tienen maravillosos resultados personales (Selva, "Las Enfermedades físicas y las emociones”; “¿Por qué un Estudio Previo antes de tomar Flores de Bach?”).

Este compromiso incondicional con la eficacia de la terapia floral corresponde a una forma de exceso de confianza que sesga los juicios acerca del rendimiento terapéutico basado en evidencias; la pretensión de que podemos manipular ciertas armonías ocultas y convergencias subyacentes entre los hechos y entidades responde cabalmente a la denominada ilusión de control (Sutherland, 1992, pp. 282-283). Por otro lado, la propensión de la terapeuta a conformar sus creencias en función de sus motivaciones y afectos puede ser caracterizada como una forma de pensamiento desiderativo o de racionalización de la esperanza, en virtud de la cual se tiende a formar creencias en la medida en que prefiere el Estado del mundo en que estas creencias se hacen verdaderas y por encima de los Estados que las hacen falsas (Elster, 1988, pp. 178-180). Asimismo, los juicios de la terapeuta en relación con la eficacia de las flores de Bach son un ejemplo palmario de sesgo confirmatorio (esto es, la consideración exclusiva y selectiva de aquellas evidencias que sostienen la creencia enunciada), pero también de sesgo a posteriori o ilusión retrospectiva (o sea, la reevaluación de nuestras historias explicativas una vez que conocemos el resultado, como si este nos resultara perfectamente predecible) (Aronson, 2000, pp. 154-155).

La terapeuta justifica la eficacia terapéutica de las flores de Bach con argumentos tan entrelazados como sesgados. Cuando no sabemos si la terapia floral puede contribuir a un tratamiento, no hay nada que perder con probar (todo lo físico se asocia a un problema interno, y la terapia es inofensiva); si la terapia floral genera problemas, se trata de una crisis curativa (que señala lo bien encaminado de la terapia); si no los genera y no hay señales de mejoría, indica el lento proceso de la terapia; si la terapia se asocia a una mejoría final (aunque solo haya complementado a otro tratamiento), se considera una demostración de que funciona. En esta extraña apuesta, nada hay que perder y, sin embargo, todo está por sanar gracias a los insondables medios de la terapia floral (Selva, "Las Crisis Curativas de Bach"; "Formas de Reacción a las Esencias Florales"). En definitiva, el discurso de la terapeuta floral lleva a cabo una neutralización de la evidencia de empeoramiento de los síntomas, como si se tratase de una crisis terapéutica pasajera que puede acompañar a la sanación, y adicionalmente neutraliza la evidencia 
de no mejoramiento, mediante la apelación al ritmo lento del proceso de una terapia profunda.

\section{Patrones de interacción en el discurso terapéutico}

Además de patentizar las estrategias argumentativas recién presentadas, el blog de terapia floral recoge en la sección "Testimonios" secuencias de interacciones discursivas bajo la forma típica de pares de turno conversacionales entre consulta y respuesta. A través de esas interacciones discursivas, se reconocen ciertos movimientos retóricos recurrentes y una determinada pauta de relación que modula las expectativas intersubjetivas y posicionamientos subjetivos de los participantes. $\mathrm{La}$ subjetivación terapéutica y la vivencia subjetiva de la terapia resultan inseparables de esos patrones de relación intersubjetiva y de la construcción retórica del marco conversacional. En las siguientes secciones examinamos los movimientos retóricos típicamente realizados en los turnos del consultante y de la tratante, para posteriormente compararlos con la pauta de interacción desarrollada en un foro de consulta médica convencional en línea.

\section{Movimientos retóricos del consultante}

En los turnos de quienes consultan a la terapeuta floral, llama la atención el carácter marcadamente testimonial de muchas intervenciones. Al privilegiar la expresión testimonial subjetiva de sus padecimientos, los consultantes refieren un profundo malestar personal que trasciende los síntomas discretos. Quien consulta no se limita a dar una descripción simple de los síntomas -asociados a algún tipo de problema psicológico de ansiedad, pánico o depresión-, sino que manifiesta una vivencia de desesperación y una marcada dependencia del apoyo de la terapeuta. Este tenor autoexpresivo de muchas de las consultas no solo enmarca la descripción de los síntomas, sino también los restantes movimientos retóricos en el turno del consultante: la caracterización del tratamiento seguido, la formulación de una consulta, la solicitud de asesoría o tratamiento y, particularmente, el agradecimiento testimonial a la terapeuta como una persona única, alguien capaz de cambiarte la vida, digna de plena confianza y sin la cual la terapia no funcionaría completamente. El dramatismo de algunos testimonios personales contrasta a veces con los tipos de consultas formuladas, frecuentemente relacionadas con la dosificación o idoneidad de alguna mezcla floral. En ese sentido, cabría pensar que -desde la perspectiva de los consultantes- la terapia alternativa reproduce a su manera el énfasis médico convencional en la medicación y en la farmacopea. No en vano se discuten los detalles, todo un ceremonial para la toma calculada de una cantidad precisa de gotas de esencias florales.

Como se aprecia en la Tabla 1, la pauta de movimientos retóricos del consultante de la terapia floral contrasta con la de quienes consultan un foro médico convencional en línea. En el paciente convencional, el tenor testimonial, el intenso vínculo interpersonal y la dependencia confiada respecto de la terapeuta dan paso a una secuencia informativa más concisa. Los participantes describen los antecedentes del caso, caracterizan sus síntomas,

\section{Tabla 1}

\section{Movimientos retóricos en los turnos del consultante}

\begin{tabular}{ll}
\hline \multicolumn{1}{c}{ Consulta médica en línea } & \multicolumn{1}{c}{ Consulta floral en línea } \\
\hline Descripción de antecedentes & Expresión de malestar personal \\
Descripción de síntomas & Descripción de síntomas \\
Descripción de indicadores & Descripción del tratamiento seguido \\
Formulación de consulta & Formulación de consulta \\
& Solicitud de tratamiento \\
& Agradecimiento personal a la terapeuta \\
\hline
\end{tabular}


describen algunos indicadores y resultados de pruebas analíticas y, finalmente, formulan una consulta concreta.

\section{Movimientos retóricos del consultado}

Los turnos de respuesta de la terapeuta floral se caracterizan por un particular compromiso subjetivo, un generoso despliegue discursivo de indicaciones para los tratamientos, justificaciones de la eficacia terapéutica de las flores de Bach y recomendaciones personales para los consultantes. Habitualmente, la terapeuta realiza los siguientes movimientos retóricos en su turno de respuesta: recomendación de tomar alguna mezcla floral, o bien instrucciones para tomar mezclas florales; recomendación de realizar un estudio personal del caso con la terapeuta; recomendación de consulta al terapeuta personal del consultante, o bien invitación a consultar personalmente con la terapeuta; invitación a consultar los artículos publicados por la terapeuta en su blog; justificación de los problemas colaterales de la terapia floral; invitación a la paciencia y constancia en el tratamiento; finalmente, algún tipo de invitación a profundizar el autoconocimiento personal, o bien alguna clase de consejo existencial. Como se puede apreciar, el enmarcado retórico de las respuestas de la terapeuta pone en primer plano la interpelación personal e individualizadora, y crea una atmósfera de seudointimidad relacional y efusión emocional. Por otra parte, el discurso de la terapeuta exhibe un tipo de organización simbólica autorreferencial y centrado en la persona del terapeuta, en virtud de la cual el consultante se ve constantemente remitido a una vinculación personal con la terapeuta y con el universo de discurso de su autoría, esto es, con los contenidos albergados en su propio blog.

En los médicos que responden consultas en línea, se da un discurso más acotado, informativo, experto y directivo. Los movimientos retóricos realizados en sus turnos de respuesta tienen las siguientes funciones: planteamiento de hipótesis diagnósticas; recomendación de consulta a un especialista; recomendación de que se realice algún tipo de prueba analítica; corrección de algunos supuestos falsos formulados por el paciente; por último, recomendación de revisar información médica de dominio público. A pesar de la amabilidad cortés en el saludo y la despedida, el tenor de las respuestas del médico suele ser impersonal, distanciado, hipotético, correctivo y especializado. Además, el modo de organización simbólica del discurso del médico convencional responde a una marcada apertura a las hipótesis alternativas, a la evidencia probatoria, al léxico disciplinar y a la voz de otros especialistas.

El discurso del médico no se enuncia desde el yo de la persona del terapeuta, como ocurre en

Tabla 2

Movimientos retóricos en los turnos de respuesta

\begin{tabular}{ll}
\hline \multicolumn{1}{c}{ Consulta médica en línea } & \multicolumn{1}{c}{ Consulta floral en línea } \\
\hline Planteamiento de hipótesis diagnósticas & Recomendación de tomar mezclas florales \\
& Instrucciones para tomar mezclas florales \\
Recomendación de realizar pruebas diagnósticas & Recomendación de estudio personal con la terapeuta \\
Recomendación de consulta a un especialista & Recomendación de consulta a su terapeuta personal \\
& Invitación a consultar personalmente con la terapeuta \\
& $\begin{array}{l}\text { Invitación a la paciencia y constancia en } \\
\text { el tratamiento }\end{array}$ \\
Corrección de los supuestos del paciente & Justificación de los problemas con la terapia \\
Recomendación de revisar información médica & Invitación a consultar los artículos contenidos en el blog de la \\
pública & terapeuta \\
& Invitación al autoconocimiento personal o consejo existencial
\end{tabular}


la terapia floral, sino que se articula con un amplio repertorio de terminología tomada del lenguaje médico, por medio de una cuidadosa modalización de los enunciados (podría tratarse de...) y mediante una comunicación directiva, que da instrucciones precisas sobre los pasos a seguir, aunque nunca prescribe un tratamiento específico por razones de ética profesional. El discurso objetivo, impersonal y profesional del médico convencional enfrenta al paciente a las opciones que hay que seguir, aunque no le prescriba la vía de tratamiento, y así se muestra respetuoso con los derechos del paciente. La terapia floral personalizada capitaliza intensamente la dependencia personal del consultante respecto de una terapeuta singular e insustituible, e introduce, paradójicamente, un patrón relacional tutelar y emocionalmente administrado.

\section{CONCLUSIÓN}

Aunque los metaanálisis disponibles muestran que la eficacia de la terapia floral no supera al placebo, es posible preguntarse por los rendimientos subjetivos de su aplicación. La eficacia subjetiva del placebo parece inseparable de cierta construcción social y del marco cultural en el que se desarrolla el discurso terapéutico, se organizan simbólicamente las expectativas y pretensiones de validez, así como se gestiona la relación interpersonal.

Los terapeutas alternativos justifican la eficacia de sus tratamientos con un vocabulario reduccionista de términos seudocientíficos y técnicas de elaboración y dosificación. Ahora bien, el efecto placebo de estos terapeutas podría sustentarse en los sesgos argumentativos, los estilos retóricos y los rituales emocionales desplegados en un discurso paraterapéutico (Goldacre, 2012, capítulos 4 y 5).

En este estudio hemos descrito cualitativamente los marcos subjetivos y los patrones de relación intersubjetiva que sustentan la comprensión del significado profundo de la terapia floral. Se han caracterizado los sesgos en el razonamiento y en la argumentación que dan forma a las estrategias de autopresentación y autojustificación de la persona del terapeuta. Particularmente, hemos identificado estrategias referidas a la personalización y autorreferencia discursiva, el saber idiográfico, la psicologización de la terapia y el error de atribución fundamental, la esencialización mistificadora y el pensamiento animista. Otras estrategias incluyen el lenguaje paraterapéutico y el léxico seudocientífico, la relativización de las opciones terapéuticas, el holismo terapéutico, así como la racionalización de la esperanza y la construcción de historias explicativas que incurren en cierta confirmación retrospectiva. Bajo la premisa de que la vivencia subjetiva de la terapia resulta inseparable de las interacciones discursivas y de los movimientos retóricos desplegados en la conversación terapéutica, hemos reconocido el tenor autoexpresivo, testimonial e intensamente personal de la terapia floral. Esa atmósfera de seudointimidad y vinculación emocional de la terapia floral se recubre de consideraciones procedimentales relativas a la preparación, dosificación y condiciones de administración de las esencias florales, como si se tratara de un ceremonial paramédico y seudofarmacológico estrictamente organizado, que así inviste de cierta aura misteriosa y falsamente científica la eficacia interpersonal de una relación paraterapéutica sujeta a cobro.

La descripción de los aspectos subjetivos e intersubjetivos resulta crucial para comprender el efecto placebo de las terapias alternativas; pero la autojustificación subjetiva de la eficacia terapéutica y los patrones interpersonales de relación en la terapia no resultan cabalmente comprendidos si no los situamos en los horizontes de sentido y en los plexos referenciales de algunas prácticas socioculturales (Thompson, Ritenbaugh y Nichter, 2010). Uno de los marcos interpretativos que permite situar adecuadamente la vivencia subjetiva de los participantes de terapias alternativas es la psicologización general de las formas de vida contemporáneas. Con justa razón, se ha señalado que el discurso psicológico resulta cada vez más decisivo en la ideología contemporánea, con todo lo que ello implica: la individualización de los procesos socioculturales, la interiorización de los problemas sociales como emociones negativas o desequilibrios mentales, la primacía de los argumentos sobre una naturaleza humana interior, la esencialización y naturalización de las diferencias personales, la patologización del malestar y el recurso a la consultoría personal, en desmedro de la transformación social estructural (Parker, 2010).

La profusión de terapias alternativas responde a cierta sensibilidad terapéutica que, desde los años setenta, desplazó a las ideologías políticas como marco de referencia de la cultura occidental, con el consiguiente repliegue a cuestiones puramente personales ligadas al bienestar personal o la autorrealización psicológica (Lasch, 1999), el autoexamen, la autenticidad seudoconfesional y a la apertura de la 
conciencia. Este ambiente terapéutico responde a un contexto de autoabsorción narcisista, de dependencia personal y de paternalismo tutelar (Lasch, 1999). El invasivo discurso terapéutico contemporáneo constituye un nuevo lenguaje del yo, que reformula profundamente los símbolos identitarios al aportar nuevos códigos para expresar y conformar la subjetivación, así como ilustra la imbricación del saber psicológico y la cultura popular en una organización simbólica para la autoexploración y la integración personal. El tipo de sujeto interpelado en la narrativa terapéutica carga de modo voluntarista con una enorme responsabilidad a la hora de acometer su autotransformación y buscar el bienestar psíquico, pero a la vez descarga a la persona de toda culpa moral por sus deficiencias (Illouz, 2010).

Esta consolidación de las retóricas psi (psicológicas y psiquiátricas) resulta funcional a las exigencias de responsabilización individual en las sociedades neoliberales y se vincula a un régimen de verdad centrado en la autoridad terapéutica (Rose, 2007). Las formas de elaboración de una ética $p s i$ pasan por la subjetivación del trabajo y la psicologización de la vida cotidiana; asimismo, convierten las competencias personales en un recurso, y proponen técnicas de autoexpresión, autoevaluación y autotransformación para lograr un pleno control de sí. El dispositivo de poder vinculado al régimen de verdad psicoterapéutico se sostiene en una relación clientelar y tutelar, mediada por el dinero, aunque heterodirigida y sacerdotal, en la medida en que la autoridad del experto terapeuta impone la pauta de la relación y el modelo de conducción de la propia vida (Rose, 2007).

Además de la cultura psicologizada y la sensibilidad terapéutica contemporánea, la vinculación subjetiva a las terapias alternativas presupone un nuevo régimen social y epistémico de la posverdad (Harsin, 2015), resultante del abandono del régimen de verdad centrado en el discurso científico, el dictamen experto y los aparatos educativos, académicos e institucionales de la sociedad moderna. El nuevo régimen socioepistémico de la posverdad se caracteriza por la circulación reticular de informaciones no verídicas, bulos y rumores, pero también por la explotación de la capacidad de autoexpresión en las redes sociales (Harsin, 2015). Este régimen de posverdad actual parece cumplir paródicamente la distopía posmoderna de una liberación de las interpretaciones divergentes y una deconstrucción radical del objetivismo. Ya no habría hechos, sino solo interpretaciones alternativas de los hechos y esquemas conceptuales inconmensurables, y solo resultaría razonable sopesar la aceptabilidad de nuestras creencias -o de los intereses locales expresados por estas-, en lugar de pretender la validez factual (Ferraris, 2012, pp. 18-22).

En términos epistemológicos, el actual régimen de la posverdad ha propiciado la difusión de un relativismo indiferentista, comprometido con cierto principio de validez equivalente, según el cual existirían muchas formas radicalmente distintas, aunque igualmente válidas, de conocer (Boghossian, 2009). Además, el relativismo epistémico posmoderno aparece frecuentemente vinculado a tesis constructivistas respecto del carácter socialmente producido de todo hecho, así como al pluralismo epistémico, caracterizado por la afirmación de que existen diferentes sistemas cognitivos, marcos de referencia y esquemas conceptuales, dependientes de circunstancias e intereses estrictamente locales (Boghossian, 2009). El antirrealismo y la denegación de la corrección factual y de la objetividad en el régimen actual de la posverdad se han traducido en una profusión sin precedentes de la charlatanería, una forma de engaño que consiste en hablar fraudulentamente sin que importen los valores veritativos (a diferencia de la mentira, que implica la enunciación deliberada de la falsedad y, por tanto, se guía negativamente por los hechos y los valores de verdad). En ese sentido, el discurso terapéutico contemporáneo de la sinceridad, la idealización del autoconocimiento transparente, la fidelidad a uno mismo y la autoexpresión sin reservas incentivarían la charlatanería, del mismo modo en que desgastan el interés en la investigación objetiva y la corrección factual (Frankfurt, 2006, pp. 76-80).

Futuras investigaciones deberán explorar qué estrategias retóricas resultan más eficientes para convencer a nuevos pacientes, así como establecer si dicha efectividad es el resultado de una interacción entre las estrategias de persuasión y las creencias previas sostenidas por la audiencia. Aún más relevante será estudiar la existencia de factores protectores que reduzcan la probabilidad de recurrir a tratamientos para los que no existe evidencia científica alguna. Es nuestro rol, como investigadores y formadores de futuros profesionales, promover el desarrollo de aprendizajes reflexivos basados en la evidencia, así como abogar por la incorporación de herramientas de pensamiento crítico y de comprensión del método científico. 


\section{REFERENCIAS}

Aronson, E. (2000). El animal social (8 ${ }^{\mathrm{a}}$ ed.). Madrid: Alianza Editorial.

Bach, E. (1936). The twelve healers and other remedies. Brightwell-cum-Sotwell, UK: The Bach Centre.

Boghossian, P. (2009). El miedo al conocimiento. Madrid: Alianza Editorial.

Callís Fernández, S. (2011). Terapia floral de Bach en niños con manifestaciones de hiperactividad. Medisan, 15 (12), 1729-1735.

Copi, I. M. (1984). Introducción a la lógica (26ª ed.). Buenos Aires: Editorial Universitaria de Buenos Aires.

Elster, J. (1988). Uvas amargas. Sobre la subversión de la racionalidad. Barcelona: Península.

Ernst, E. (2010). Bach flower remedies: a systematic review of randomised clinical trials. Swiss Medical Weekly, 140, w13079, http://doi.org/10.4414/smw.2010.13079

Escandell Vidal, M. V. (2014). La comunicación. Lengua, cognición y sociedad. Madrid: Akal.

Ferraris, M. (2012). Manifiesto del nuevo realismo. Santiago de Chile: Ariadna Ediciones.

Frankfurt, H. G. (2006). On Bullshit. Sobre la manipulación de la verdad. Barcelona: Paidós.

Goldacre, B. (2012). Mala ciencia. Barcelona: Planeta.

Halberstein, R. A., Sirkin, A., y Ojeda-Vaz, M. M. (2010). When less is better: a comparison of Bach Flower Remedies and homeopathy. Annals of Epidemiology, 20 (4), 298-307, http:// doi.org/10.1016/j.annepidem.2009.11.006

Hamblin, C. L. (2016). Falacias. Lima: Palestra.

Harsin, J. (2015). Regimes of Posttruth, Postpolitics, and Attention Economies. Communication, Culture \& Critique, 8, 327-333.

Howard, J. (2007). Do Bach flower remedies have a role to play in pain control? A critical analysis investigating therapeutic value beyond the placebo effect, and the potential of Bach flower remedies as a psychological method of pain relief. Complementary Therapies in Clinical Practice, 13, 174-183. DOI: 10.1016/j.ctcp.2007.03.001

Illouz, E. (2010). La salvación del alma moderna. Buenos Aires: Katz.

Lack, C. W., y Rousseau, J. (2016). Critical thinking, science, and pseudoscience. New York: Springer.

Lakoff, G., y Johnson, M. (1998). Metáforas de la vida cotidiana ( $4^{\mathrm{a}}$ ed.). Madrid: Cátedra.

Lasch, C. (1999). La cultura del narcisismo. Santiago de Chile: Andrés Bello.

López Cantos, F. (2017a). Comunicación pública de la pseudociencia: homeópatas y orgonitas 2.0. Razón y palabra, 21 (96), 355-372. Recuperado de https://www.revistarazonypalabra.org/ index.php/ryp/article/view/836

López Cantos, F. (2017b). El discurso de la felicidad de las terapias alternativas en Facebook. Razón y palabra, 21 (98), 381-393. Recuperado de http://ww.revistarazonypalabra.com/ index.php/ryp/article/view/953
Mantle, F. (1997). Bach flower remedies. Complementary Therapies in Nursing \& Midwifery, 3 (5), 142-144, http://doi. org/10.1016/S1353-6117(97)80015-7

Marraud, H. (2007). Methodus argumentandi. Madrid: Ediciones de la Universidad Autónoma de Madrid.

Marraud,H.(2013).¿Eslógic@?Análisis yevaluación de argumentos. Madrid: Cátedra.

Parker, I. (2010). La psicología como ideología. Madrid: Catarata.

Pintov, S., Hochman, M., Livne, A., Heyman, E., y Lahat, E. (2005). Bach flower remedies used for attention deficit hyperactivity disorder in children--a prospective double blind controlled study. European Journal of Paediatric Neurology, 9 (6), 395-398, http://doi.org/10.1016/j.ejpn.2005.08.001

Plantin, C. (2011). La argumentación. Barcelona: Ariel.

Pohl, R. (Ed.) (2004). Cognitive illusions. New York: Psychology Press.

Popper, K. (1984). La miseria del historicismo. Madrid: Alianza Editorial.

Potter, J. (1998). La representación de la realidad. Discurso, retórica y construcción social. Barcelona: Paidós.

Rescher, N. (1993). La racionalidad. Madrid: Tecnos.

Resende, M. M. de C., Costa, F. E. de C., Gardona, R. G. B., Araújo, R. G., Mundim, F. G. L., y Costa, M. J. de C. (2014). Preventive use of Bach flower Rescue Remedy in the control of risk factors for cardiovascular disease in rats. Complementary Therapies in Medicine, 22 (4), 719-723, http://doi.org/10.1016/j. ctim.2014.06.008

Rose, N. (2007). Terapia y poder: techné y ethos. Archipiélago, 76, 101-124.

Rossi-Landi, F. (1980). Ideología. Barcelona: Labor.

Selva, A. (2010-2018). Gotas de Bach [Blog]. Recuperado de: http://www.gotasdebach.com

Shotter, J. (2001). Realidades conversacionales. La construcción de la vida a través del lenguaje. Buenos Aires: Amorrortu.

Sokal, A., y Bricmont, J. (1999). Imposturas intelectuales. Barcelona: Paidós.

Strauss, A., y Corbin, J., (2002). Bases de la investigación cualitativa. Antioquía, Colombia: Editorial Universidad de Antioquía.

Sutherland, S. (1992). La irracionalidad. El enemigo interior. Madrid: Alianza Editorial.

Swales, J. M. (2004). Research Genres. Cambridge: Cambridge University Press.

Taylor, S. J., y Bogdan, R. (1994). Introducción a los métodos cualitativos de investigación. Barcelona: Paidós.

Thaler, K., Kaminski, A., Chapman, A., Langley, T., y Gartlehner, G. (2009). Bach Flower Remedies for psychological problems and pain: a systematic review. BMC Complementary and Alternative Medicine, 9 (1), 16, http://doi.org/10.1186/1472-6882-9-16

Thompson, J. J., Ritenbaugh, C., y Nichter, M. (2010). Reconsidering the Placebo Response from a Broad Anthropological 
Perspective. Culture, Medicine and Psychiatry, 33 (1),112-152. DOI: $10.1007 / \mathrm{s} 11013-008-9122-2$

Tindale, C. W. (2007). Fallacies and argument appraisal. New York: Cambridge University Press.

Tversky, A., y Kahneman, D. (1974). Judgment under Uncertainty: Heuristics and Biases. Science, 185 (4157), 1124-1131, https:// doi.org/10.1126/science.185.4157.1124

Vega Reñón, L. (2013). La fauna de las falacias. Madrid: Trotta. Walach, H., Rilling, C., y Engelke, U. (2001). Efficacy of Bachflower remedies in test anxiety: a double-blind, placebo-controlled, randomized trial with partial crossover. Journal of Anxiety Disorders, 15 (4), 359-366.

Walton, D. N. (1999). One-sided arguments. New York: State University of New York.

Walton, D. N., y Krabbe, E. C. W. (2017). Argumentación y normatividad dialógica. Compromisos y razonamiento interpersonal. Lima: Palestra.

Wilson, D., y Sperber, D. (2004). La teoría de la relevancia. Revista de Investigación Lingüística, 7 (1), 237-286. 